\title{
A NEW APPROACH FOR THE APPROXIMATE SOLUTION OF FRACTIONAL INTEGRO-DIFFERENTIAL EQUATIONS
}

\author{
MINE AYLIN BAYRAK ${ }^{a}$ AND ALI DEMIR ${ }^{b}$ \\ Department of Mathematics, Kocaeli University, Izmit, Kocaeli, Turkey. \\ ${ }^{a}$ E-mail: aylin@kocaeli.edu.tr \\ ${ }^{b}$ E-mail: ademir@kocaeli.edu.tr
}

\|\|$\|$

Abstract

In this paper, the construction of the analytical solutions of space-time fractional integro-differential equations are studied. We first transform the space-time fractional integro-differential equations into space-time fractional differential equations by means of new transformations then construct the solutions via residual power series method (RPSM). Finally, we give some examples to illustrate how powerful and effective the method is.

\section{Introduction}

Since the mathematical models including fractional derivative are more realistic then the one including integer order derivatives, the concept of fractional calculus gets increasing attention from many scientists. As a result, this concept has various applications in many fields such as mathematics, physics and engineering [1, 22, 23, 24]. By means of nonsingular and singular kernels, quite a few fractional derivatives are defined to model engineering and scientific phenomena better [7, 8, 9, 10, 11]. Since obtaining an analytical solution of fractional integro-differential equations is impossible or inconvenient, various numerical methods have been developed to construct the solutions of them [1, 2, 5, 6, 12, 13, 14, 15, 16, 17, 21, 25, 26].

Received September 9, 2021.

AMS Subject Classification: 35R11.

Key words and phrases: Residual power series method, space-time fractional integro-differential equations, Caputo derivative. 
RPSM, developed by the Jordan mathematician Abu Arqub [2], is a very useful analytical method to establish the solutions of different types of FPDEs in series form $[3,4,4,20,21,22,23,6,8,29]$.

In this manuscript, we consider the following space-time fractional integrodifferential equations:

$$
\begin{gathered}
D_{t}^{\alpha} u=I_{x}^{\beta} u+F(x, t, u), \\
u(x, 0)=\psi(x), \quad 0<x \leq l, \\
u(0, t)=\mu_{1}(t), \quad 0<t \leq T, \\
u_{x}(0, t)=\mu_{2}(t), \quad 0<t \leq T,
\end{gathered}
$$

where $0 \leq n<\alpha \leq n+1,0 \leq m<\beta \leq m+1$. The RPSM is implemented to construct a new algorithm for determining analytical solutions of the problem above. The existence and uniqueness as well as convergence of the approximate solution of this problem is proved in [27].

\section{Preliminaries}

We first give the main definitions and various features of the fractional calculus theory in this section. The Riemann-Liouville fractional integral operator of order $\alpha(\alpha \geq 0)$ is defined as

$$
\begin{aligned}
J^{\alpha} f(x) & =\frac{1}{\Gamma(\alpha)} \int_{0}^{x}(x-t)^{\alpha-1} f(t) d t, \alpha>0, x>0, \\
J^{0} f(x) & =f(x) .
\end{aligned}
$$

The Caputo fractional derivative of order $\alpha$ is defined as

$$
\begin{array}{r}
D^{\alpha} f(x)=J^{m-\alpha} D^{m} f(x)=\frac{1}{\Gamma(m-\alpha)} \int_{0}^{x} \begin{array}{l}
(x-t)^{m-\alpha-1} f^{(m)}(t) d t, \\
m-1<\alpha \leq m, x>0,
\end{array}
\end{array}
$$

where $D^{m}$ is the classical differential operator of order $m$.

The time fractional derivative operator of order $\alpha$ of $u(x, t)$ is defined 
as [18, 19]

$$
\begin{aligned}
D_{t}^{\alpha} u(x, t) & =\frac{\partial^{\alpha} u(x, t)}{\partial t^{\alpha}} \\
& = \begin{cases}\frac{1}{\Gamma(n-\alpha)} \int_{0}^{t}(t-\tau)^{n-\alpha-1} \frac{\partial^{n} u(\tau, t)}{\partial t^{n}} d \tau, & n-1<\alpha \leq n, \\
\frac{\partial^{n} u(x, t)}{\partial t^{n}}, & \alpha=n \in N,\end{cases}
\end{aligned}
$$

where $n$ is the smallest integer greater than $\alpha$.

\section{Solving the Fractional Integro-Differential Equations (FIE)}

In this section, we achieve the solution of fractional integro-differential equations (11) - (2) with the help of the transformation $u=D_{x}^{\beta} v$ and RPSM. We consider the following two cases.

Case 1: Solution of FIE by the transformation $u=D_{x}^{\beta} v$ for $n=m=0$

By the help of the transformation $u=D_{x}^{\beta} v$, we eliminate the RiemannLiouville integration in Eq. (10). As a result, the following problem is obtained:

$$
\begin{aligned}
D_{t}^{\alpha}\left(D_{x}^{\beta} v\right) & =v-\frac{I_{x}^{\beta} \mu_{1}(t)}{\Gamma(2-\beta) \Gamma(\beta)}+F\left(x, t, D_{x}^{\beta} v\right), \\
v(x, 0) & =I_{x}^{\beta} \psi(x), \quad 0<x \leq 1, \\
v(0, t) & =I_{x}^{\beta} \mu_{1}(t), \quad 0<t \leq 1 .
\end{aligned}
$$

By the initial condition (10), the $0^{t h}$-RPS approximate solution $v_{0}(x, t)$ is established as follows

$$
v_{0}(x, t)=v(x, 0)=I_{x}^{\beta} \psi(x)=f_{0}(x)
$$

and $k^{t h}$-truncated residual function of Eq. (9) is

$$
\begin{array}{r}
\operatorname{Res}_{k}=D_{t}^{\alpha}\left(D_{x}^{\beta} v_{k}\right)-v_{k}+I_{x}^{\beta} \mu_{1}(t) \frac{1}{\Gamma(2-\beta) \Gamma(\beta)}-F\left(x, t, D_{x}^{\beta} v_{k}\right), \\
k=0,1,2, \ldots
\end{array}
$$


Now the steps below are taken:

Step 1: To construct $f_{1}(x)$, substituting

$$
v_{1}(x, t)=f_{0}(x)+f_{1}(x) \frac{t^{\alpha}}{\Gamma(1+\alpha)}
$$

into Eq. (13) leads to

$\operatorname{Res}_{1}=D_{x}^{\beta} f_{1}(x)-f_{0}(x)-f_{1}(x) \frac{t^{\alpha}}{\Gamma(1+\alpha)}+I_{x}^{\beta} \mu_{1}(t) \frac{1}{\Gamma(2-\beta) \Gamma(\beta)}-F$.

plugging $t=0$, we have the following results:

$$
f_{1}(0)=I_{x}^{2 \beta} \mu_{1}(0), \quad f_{1}(x)=I_{x}^{\beta} f_{0}(x)+\left.I_{x}^{\beta} F\right|_{t=0} .
$$

Step 2: To establish $f_{2}(x)$, we substitute

$$
v_{2}(x, t)=f_{0}(x)+f_{1}(x) \frac{t^{\alpha}}{\Gamma(1+\alpha)}+f_{2}(x) \frac{t^{2 \alpha}}{\Gamma(1+2 \alpha)},
$$

into Eq. (13) and use

$$
\begin{aligned}
\operatorname{Res}_{2}= & D_{x}^{\beta} f_{1}(x)+D_{x}^{\beta} f_{2}(x) \frac{t^{\alpha}}{\Gamma(1+\alpha)}-f_{0}(x)-f_{1}(x) \frac{t^{\alpha}}{\Gamma(1+\alpha)} \\
& -f_{2}(x) \frac{t^{2 \alpha}}{\Gamma(1+2 \alpha)}+I_{x}^{\beta} \mu_{1}(t) \frac{1}{\Gamma(2-\beta) \Gamma(\beta)}-F
\end{aligned}
$$

in Eq. (13). Hence $f_{2}(x)$ becomes

$$
f_{2}(0)=I_{x}^{2 \beta} D_{t}^{\alpha} \mu_{1}(0), \quad f_{2}(x)=I_{x}^{\beta} f_{1}(x)+\left.I_{x}^{\beta} D_{t}^{\alpha} F\right|_{t=0},
$$

and so on.

As a result, the solution of (91)-(11) can be acquired in the following form:

$$
\begin{aligned}
v(x, t)= & f_{0}(x)+\left(I_{x}^{\beta} f_{0}(x)+\left.I_{x}^{\beta} F\right|_{t=0}\right) \frac{t^{\alpha}}{\Gamma(1+\alpha)} \\
& +\left(I_{x}^{\beta} f_{1}(x)+\left.I_{x}^{\beta} D_{t}^{\alpha} F\right|_{t=0}\right) \frac{t^{2 \alpha}}{\Gamma(1+2 \alpha)}+\cdots
\end{aligned}
$$

By the help of the transformation $u(x, t)=D_{x}^{\beta} v(x, t)$, the solution of problem 
(11) - (3) is constructed in the following form

$$
\begin{aligned}
u(x, t)= & \psi(x)+\left(I_{x}^{\beta} \psi(x)+\left.F\right|_{t=0}\right) \frac{t^{\alpha}}{\Gamma(1+\alpha)} \\
& +\left(I_{x}^{2 \beta} \psi(x)+\left.I_{x}^{\beta} F\right|_{t=0}+\left.D_{t}^{\alpha} F\right|_{t=0}\right) \frac{t^{2 \alpha}}{\Gamma(1+2 \alpha)}+\cdots
\end{aligned}
$$

Case 2: Solution of the FIE by the transformation $u=D_{x}^{\beta} v$ for $n=0$ and $m=1$

By taking the transformation $u=D_{x}^{\beta} v$ into account, we eliminate the Riemann-Liouville integration in Eq. (1). As a result, the following problem is obtained

$$
\begin{gathered}
D_{t}^{\alpha}\left(D_{x}^{\beta} v\right)=v-\frac{I_{x}^{\beta} \mu_{2}(t) x}{\Gamma(3-\beta) \Gamma(\beta)}-\frac{I_{x}^{\beta} \mu_{1}(t)}{\Gamma(3-\beta) \Gamma(\beta-1)}+F\left(x, t, D_{x}^{\beta} v\right), \\
v(x, 0)=I_{x}^{\beta} \psi(x), \quad 0<x \leq 1, \\
v(0, t)=I_{x}^{\beta} \mu_{1}(t), \quad 0<t \leq 1, \\
v_{x}(0, t)=I_{x}^{\beta} \mu_{2}(t), \quad 0<t \leq 1 .
\end{gathered}
$$

The $k^{\text {th }}$-truncated residual function of Eq. (22) is obtained in the following form

$$
\begin{aligned}
R e s_{k}= & D_{t}^{\alpha}\left(D_{x}^{\beta} v_{k}\right)-v_{k}+\frac{I_{x}^{\beta} \mu_{2}(t) x}{\Gamma(3-\beta) \Gamma(\beta)}+\frac{I_{x}^{\beta} \mu_{1}(t)}{\Gamma(3-\beta) \Gamma(\beta-1)} \\
& -F\left(x, t, D_{x}^{\beta} v_{k}\right), \quad k=0,1,2, \ldots
\end{aligned}
$$

As above, following steps are taken :

Step 1: By the help of the initial condition, initial approximation becomes

$$
v_{0}(x, t)=I_{x}^{\beta} \psi(x)=f_{0}(x)
$$

In order to construct $f_{1}(x)$, we substitute

$$
v_{1}(x, t)=f_{0}(x)+f_{1}(x) \frac{t^{\alpha}}{\Gamma(1+\alpha)},
$$


into Eq. (26) which leads to the following

$$
\begin{aligned}
R e s_{1}= & D_{x}^{\beta} f_{1}(x)-f_{0}(x)-f_{1}(x) \frac{t^{\alpha}}{\Gamma(1+\alpha)}-f_{2}(x) \frac{t^{2 \alpha}}{\Gamma(1+2 \alpha)} \\
& -\frac{I_{x}^{\beta} \mu_{2}(t) x}{\Gamma(3-\beta) \Gamma(\beta)}-\frac{I_{x}^{\beta} \mu_{1}(t)}{\Gamma(3-\beta) \Gamma(\beta-1)}-F .
\end{aligned}
$$

As a result, we have

$$
f_{1}(0)=I_{x}^{2 \beta} \mu_{1}(0), \quad f_{1}^{\prime}(0)=I_{x}^{2 \beta} \mu_{2}(0), \quad f_{1}(x)=I_{x}^{\beta} f_{0}(x)+\left.I_{x}^{\beta} F\right|_{t=0} .
$$

Step 2: To construct $f_{2}(x)$, plugging

$$
v_{2}(x, t)=f_{0}(x)+f_{1}(x) \frac{t^{\alpha}}{\Gamma(1+\alpha)}+f_{2}(x) \frac{t^{2 \alpha}}{\Gamma(1+2 \alpha)},
$$

into Eq. (26) which leads to the following results

$$
f_{2}(0)=I_{x}^{2 \beta} D_{t}^{\alpha} \mu_{1}(0), f_{2}^{\prime}(0)=I_{x}^{2 \beta} D_{t}^{\alpha} \mu_{2}(0), f_{2}(x)=I_{x}^{\beta} f_{1}(x)+\left.I_{x}^{\beta} D_{t}^{\alpha} F\right|_{t=0},
$$

and so on.

The solution of (22) - (25) is obtained in the series form as follows:

$$
\begin{aligned}
v(x, t)= & f_{0}(x)+\left(I_{x}^{\beta} f_{0}(x)+\left.I_{x}^{\beta} F\right|_{t=0}\right) \frac{t^{\alpha}}{\Gamma(1+\alpha)} \\
& +\left(I_{x}^{\beta} f_{1}(x)+\left.I_{x}^{\beta} D_{t}^{\alpha} F\right|_{t=0}\right) \frac{t^{2 \alpha}}{\Gamma(1+2 \alpha)}+\cdots
\end{aligned}
$$

Finally, the solution of (11) - (4) is constructed in the following form via the transformation $u(x, t)=D_{x}^{\beta} v(x, t)$

$$
\begin{aligned}
u(x, t)= & \psi(x)+\left(I_{x}^{\beta} \psi(x)+\left.F\right|_{t=0}\right) \frac{t^{\alpha}}{\Gamma(1+\alpha)} \\
& +\left(I_{x}^{2 \beta} \psi(x)+\left.I_{x}^{\beta} F\right|_{t=0}+\left.D_{t}^{\alpha} F\right|_{t=0}\right) \frac{t^{2 \alpha}}{\Gamma(1+2 \alpha)}+\cdots
\end{aligned}
$$

\section{Numerical Examples}

In this section, some examples are presented to illustrate the obtained results in Case 1 and Case 2. 
Example 1. Let us consider the problem including FIE

$$
\begin{aligned}
D_{t}^{\alpha} u & =I_{x}^{\beta} u, 0<\alpha, \beta \leq 1 \\
u(x, 0) & =\exp (2 x), \\
u(0, t) & =\exp \left(\frac{t}{2}\right),
\end{aligned}
$$

which has the exact solution $u(x, t)=\exp \left(2 x+\frac{t}{2}\right)$. Eqs. (35)-(37) are transformed as follows:

$$
\begin{aligned}
D_{t}^{\alpha}\left(D_{x}^{\beta} v\right) & =v-\frac{I_{x}^{\beta} \exp \left(\frac{t}{2}\right)}{\Gamma(2-\beta) \Gamma(\beta)}, \\
v(x, 0) & =I_{x}^{\beta} \exp (2 x)=f_{0}(x) . \\
v(0, t) & =I_{x}^{\beta} \exp \left(\frac{t}{2}\right) .
\end{aligned}
$$

The $k^{\text {th }}$-truncated residual function is obtained in the following form

$$
\operatorname{Res}_{k}=D_{t}^{\alpha}\left(D_{x}^{\beta} v_{k}\right)-v_{k}+\frac{I_{x}^{\beta} \exp \left(\frac{t}{2}\right)}{\Gamma(2-\beta) \Gamma(\beta)}, k=0,1,2, \ldots
$$

Based on the RPS procedure, the first two coefficients are obtained as follows:

$$
\begin{aligned}
& f_{1}(x)=I_{x}^{\beta} \exp (2 x), \\
& f_{2}(x)=I_{x}^{2 \beta} \exp (2 x),
\end{aligned}
$$

and so on.

Hence the approximate solution of (35) - (37) is constructed in the following form:

$$
\begin{aligned}
u(x, t)= & \exp (2 x)+\frac{x^{1+\beta}}{2} E_{1,2+\beta}(2 x) \frac{t^{\alpha}}{\Gamma(1+\alpha)} \\
& +\frac{x^{2+2 \beta}}{4} E_{1,3+2 \beta}(2 x) \frac{t^{2 \alpha}}{\Gamma(1+2 \alpha)}+\cdots
\end{aligned}
$$

From Table 1 and Figures $1-3$, it is clear that as $\alpha$ and $\beta$ increase to 1 the solution gets closer to the exact solution of FIE with integer orders for all values of $x$ and $t$. 


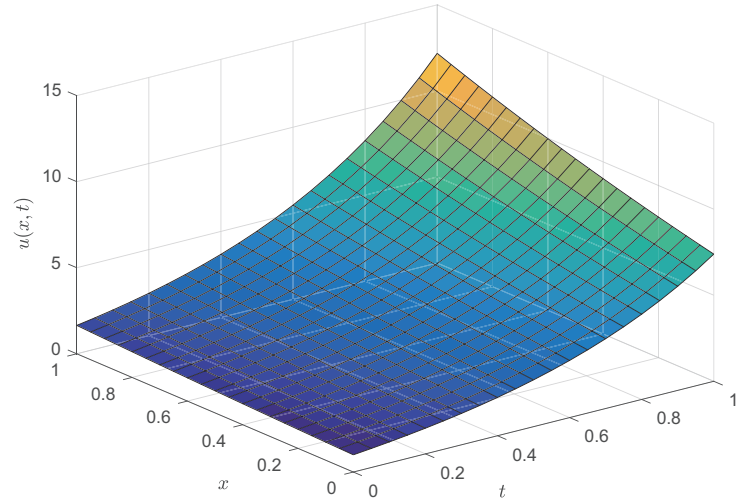

Figure 1: The exact solution of Ex. 1.

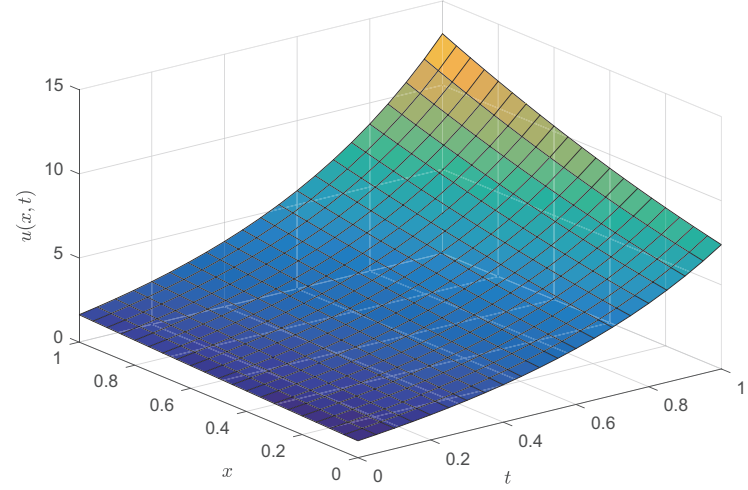

Figure 2: The RPS solution of Ex. 1 for $\alpha=1, \beta=1$.

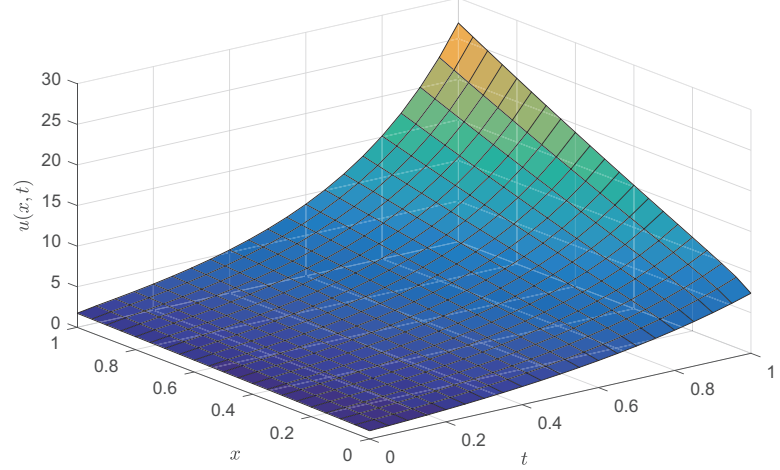

Figure 3: The RPS solution of Ex. 1 for $\alpha=0.75, \beta=0.75$. 
Table 1: The values of the approximate solutions for Example 1.

\begin{tabular}{|c|c|c|ccc|rrr|}
\hline$x$ & $t$ & Exact & \multicolumn{3}{|c|}{$\alpha=1$} & \multicolumn{3}{c|}{$\alpha=0.75$} \\
& & & $\beta=1$ & $\beta=0.75$ & $\beta=0.5$ & $\beta=1$ & $\beta=0.75$ & $\beta=0.5$ \\
\hline 0.2 & 0.3 & 1.73325 & 1.74241 & 1.80249 & 1.89331 & 1.92097 & 2.02992 & 2.18494 \\
& 0.6 & 2.01375 & 2.04664 & 2.18736 & 2.39971 & 2.30467 & 2.52219 & 2.83174 \\
& 0.9 & 2.33965 & 2.40449 & 2.64645 & 3.01103 & 2.70182 & 3.03740 & 3.51498 \\
0.4 & 0.3 & 2.58571 & 2.60720 & 2.70573 & 2.83398 & 2.96675 & 3.16899 & 3.42195 \\
& 0.6 & 3.00417 & 3.08450 & 3.31304 & 3.60951 & 3.65261 & 4.05166 & 4.54979 \\
& 0.9 & 3.49034 & 3.65746 & 4.04747 & 4.55212 & 4.37429 & 4.98516 & 5.74672 \\
0.6 & 0.3 & 3.85743 & 3.89509 & 4.03828 & 4.21271 & 4.63828 & 4.99661 & 5.43093 \\
& 0.6 & 4.48169 & 4.62396 & 4.95325 & 5.35278 & 5.87339 & 6.57382 & 7.42059 \\
& 0.9 & 5.20698 & 5.50674 & 6.06502 & 6.74034 & 7.18055 & 8.24612 & 9.53207 \\
0.8 & 0.3 & 5.75460 & 5.81418 & 6.01686 & 6.25631 & 7.38653 & 8.04989 & 8.85895 \\
& 0.6 & 6.68589 & 6.91171 & 7.37465 & 7.91950 & 9.67309 & 10.96190 & 12.53057 \\
& 0.9 & 7.76790 & 8.24563 & 9.02642 & 9.94261 & 12.09615 & 14.04878 & 16.42211 \\
\hline
\end{tabular}

Example 2. Let us consider the problem including FIE

$$
\begin{gathered}
D_{t}^{\alpha} u=I_{x}^{\beta} u+\left(1-\frac{x}{2}\right) u, 0<\alpha, \beta \leq 1, \\
u(x, 0)=2 x \\
u(0, t)=0
\end{gathered}
$$

which has the exact solution $u(x, t)=2 x \exp (t)$. Eqs. (43)-(45) are transformed as follows:

$$
\begin{aligned}
D_{t}^{\alpha}\left(D_{x}^{\beta} v\right) & =v+\left(1-\frac{x}{2}\right) D_{x}^{\beta} v, \\
v(x, 0) & =I_{x}^{\beta}(2 x)=f_{0}(x), \\
v(0, t) & =0 .
\end{aligned}
$$

The $k^{\text {th }}$-truncated residual function is obtained in the following form

$$
\operatorname{Res}_{k}=D_{t}^{\alpha}\left(D_{x}^{\beta} v_{k}\right)-v_{k}-\left(1-\frac{x}{2}\right) D_{x}^{\beta} v_{k}, k=0,1,2, \ldots
$$

Based on the RPS procedure the first two coefficients are obtained as follows:

$$
\begin{aligned}
& f_{1}(x)=I_{x}^{\beta} f_{0}(x)-\left(1-\frac{x}{2}\right) D_{x}^{\beta} f_{0}(x), \\
& f_{2}(x)=I_{x}^{\beta} f_{1}(x)-\left(1-\frac{x}{2}\right) D_{x}^{\beta} f_{1}(x),
\end{aligned}
$$


Table 2: The values of approximate solutions for Example 2.

\begin{tabular}{|c|c|c|c|c|c|c|c|c|}
\hline \multirow{2}{*}{$x$} & \multirow{2}{*}{$t$} & \multirow{2}{*}{ Exact } & \multicolumn{3}{|c|}{$\alpha=1$} & \multicolumn{3}{|c|}{$\alpha=0.75$} \\
\hline & & & $\beta=1$ & $\beta=0.75$ & $\beta=0.5$ & $\beta=1$ & $\beta=0.75$ & $\beta=0.5$ \\
\hline \multirow[t]{3}{*}{0.2} & 0.3 & 0.53994 & 0.55420 & 0.57743 & 0.61513 & 0.67037 & 0.72102 & 0.80152 \\
\hline & 0.6 & 0.72885 & 0.77680 & 0.84911 & 0.96379 & 0.96242 & 1.08830 & 1.28554 \\
\hline & 0.9 & 0.98384 & 1.06780 & 1.21502 & 1.44597 & 1.29029 & 1.50927 & 1.85013 \\
\hline \multirow[t]{3}{*}{0.4} & 0.3 & 1.07989 & 1.10480 & 1.14946 & 1.21161 & 1.33084 & 1.41897 & 1.53975 \\
\hline & 0.6 & 1.45770 & 1.53920 & 1.66372 & 1.83409 & 1.89686 & 2.10052 & 2.37617 \\
\hline & 0.9 & 1.96768 & 2.10320 & 2.34279 & 2.66742 & 2.52921 & 2.87113 & 3.33092 \\
\hline \multirow[t]{3}{*}{0.6} & 0.3 & 1.61983 & 1.65180 & 1.71639 & 1.79757 & 1.98143 & 2.10192 & 2.25190 \\
\hline & 0.6 & 2.18654 & 2.28720 & 2.45638 & 2.66673 & 2.80334 & 3.06897 & 3.39680 \\
\hline & 0.9 & 2.95152 & 3.10620 & 3.41997 & 3.80749 & 3.71674 & 4.15163 & 4.68577 \\
\hline \multirow[t]{3}{*}{0.8} & 0.3 & 2.15977 & 2.19520 & 2.27641 & 2.37008 & 2.62213 & 2.76803 & 2.93530 \\
\hline & 0.6 & 2.91539 & 3.02080 & 3.22475 & 3.45839 & 3.68185 & 3.99262 & 4.34686 \\
\hline & 0.9 & 3.93536 & 4.07680 & 4.44501 & 4.86494 & 4.85288 & 5.35180 & 5.91860 \\
\hline
\end{tabular}

and so on.

Hence the approximate solution of (43) - (45) is constructed in the following form:

$$
\begin{aligned}
u(x, t)= & 2 x+\left(2 \frac{x^{1+\beta}}{\Gamma(2+\beta)}+2 x-x^{2}\right) \frac{t^{\alpha}}{\Gamma(1+\alpha)}+\left(2 \frac{x^{1+2 \beta}}{\Gamma(2+2 \beta)}+2 \frac{x^{\beta}}{\Gamma(1+\beta)}\right. \\
& \left.-2 \frac{x^{2+\beta}}{\Gamma(3+\beta)}+(2-x) \frac{x^{1+\beta}}{\Gamma(2+\beta)}+2 x-2 x^{2}+\frac{x^{3}}{2}\right) \frac{t^{2 \alpha}}{\Gamma(1+2 \alpha)}+\cdots .
\end{aligned}
$$

From Table 2 and Figures $4-6$, it is clear that as $\alpha$ and $\beta$ increase to 1 the solution gets closer to the exact solution of FIE with integer orders for all values of $x$ and $t$. However as $t$ increases, the solution gets away from the exact solution of FIE with integer orders. 


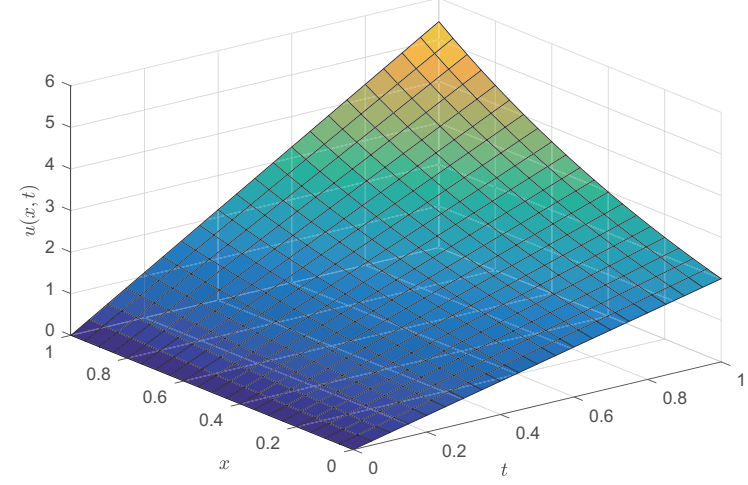

Figure 4: The exact solution of Ex. 2.

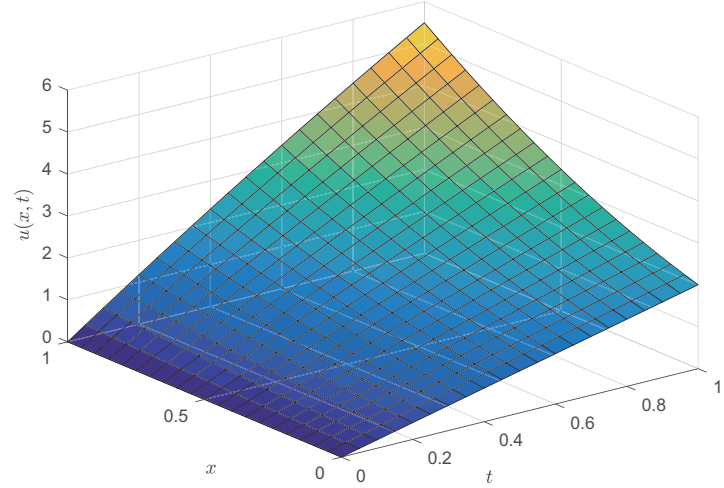

Figure 5: The RPS solution of Ex. 2 for $\alpha=1, \beta=1$.

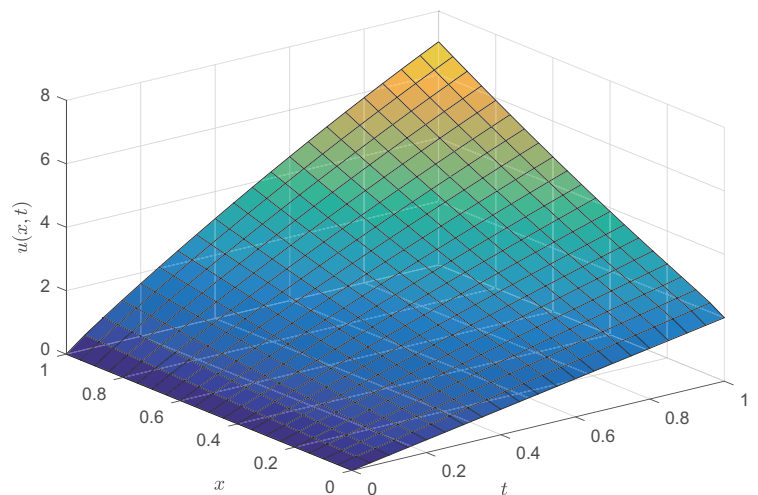

Figure 6: The RPS solution of Ex. 2 for $\alpha=0.75, \beta=0.75$. 
Example 3. Let us consider the problem including FIE

$$
\begin{aligned}
D_{t}^{\alpha} u= & I_{x}^{\beta} u+u^{2}-4 x(1-4 t)-x^{2}\left(5-18 t+52 t^{2}-64 t^{3}+64 t^{4}\right), 0<\alpha, \beta \leq 1 \\
& u(x, 0)=2 x \\
& u(0, t)=0
\end{aligned}
$$

which has the exact solution $u(x, t)=2 x\left(1-2 t+4 t^{2}\right)$. Applying transformations to Eqs. (51)-(53), we have :

$$
\begin{gathered}
D_{t}^{\alpha}\left(D_{x}^{\beta} v\right)=v+\left(D_{x}^{\beta} v\right)^{2}-4 x(1-4 t)-x^{2}\left(5-18 t+52 t^{2}-64 t^{3}+64 t^{4}\right), \\
v(x, 0)=2 \frac{x^{1+\beta}}{\Gamma(2+\beta)}=f_{0}(x), \\
v(0, t)=0 .
\end{gathered}
$$

The $k^{\text {th }}$-truncated residual function becomes

$$
\operatorname{Res}_{k}=D_{t}^{\alpha}\left(D_{x}^{\beta} v_{k}\right)-v_{k}-D_{x}^{\beta} v D_{x}^{\beta+1} v+\frac{x^{2}}{1-2 t}, k=0,1,2, \ldots
$$

Based on the RPS procedure, the first two coefficients are obtained as follows:

$$
\begin{aligned}
f_{1}(x)= & 2 \frac{x^{1+2 \beta}}{\Gamma(2+2 \beta)}+8 \frac{x^{2+\beta}}{\Gamma(3+\beta)}-4 \frac{x^{1+\beta}}{\Gamma(2+\beta)}-10 \frac{x^{2+\beta}}{\Gamma(3+\beta)}, \\
f_{2}(x)= & 2 \frac{x^{1+3 \beta}}{\Gamma(2+3 \beta)}-2 \frac{x^{2+2 \beta}}{\Gamma(3+2 \beta)}-4 \frac{x^{1+2 \beta}}{\Gamma(2+2 \beta)}+8 \frac{\Gamma(3+\beta)}{\Gamma(2+\beta)} \frac{x^{2+2 \beta}}{\Gamma(3+2 \beta)} \\
& -8 \frac{x^{3+\beta}}{\Gamma(4+\beta)}-32 \frac{x^{2+\beta}}{\Gamma(3+\beta)},
\end{aligned}
$$

and so on.

Hence the approximate solution of (51) - (53) is constructed in the following form:

$$
\begin{aligned}
u(x, t)= & 2 x+\left(2 \frac{x^{1+\beta}}{\Gamma(2+\beta)}-4 x-x^{2}\right) \frac{t^{\alpha}}{\Gamma(1+\alpha)}+\left(2 \frac{x^{1+2 \beta}}{\Gamma(2+2 \beta)}-2 \frac{x^{2+\beta}}{\Gamma(3+\beta)}\right. \\
& \left.-4 \frac{x^{1+\beta}}{\Gamma(2+\beta)}+8 \frac{x^{2+\beta}}{\Gamma(3+\beta)}-4 \frac{x^{3}}{3}-16 x^{2}\right) \frac{t^{2 \alpha}}{\Gamma(1+2 \alpha)}+\cdots
\end{aligned}
$$

From Table 3 and Figures $7-9$, it is clear that as $\alpha$ and $\beta$ increase to 1 the solution gets closer to the exact solution of FIE with integer orders for all values of $x$. However as $t$ increases, the solution is get away from the exact solution of FIE with integer orders. 


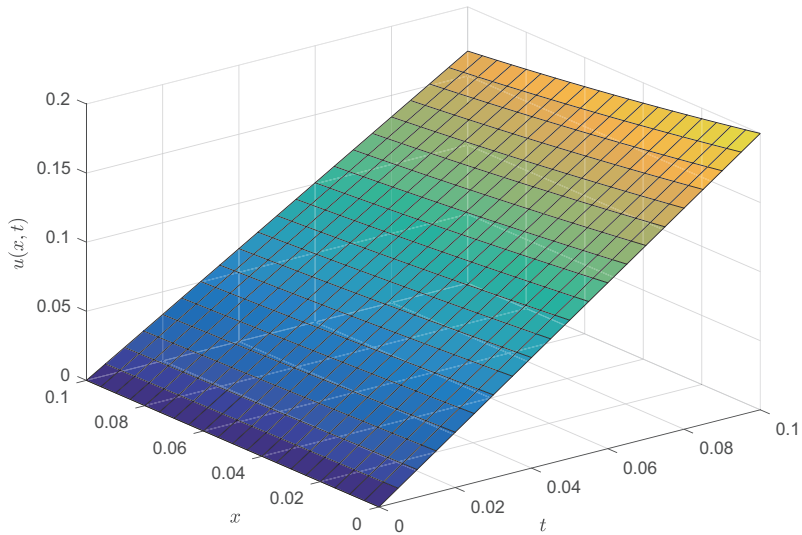

Figure 7: The exact solution of Ex. 3.

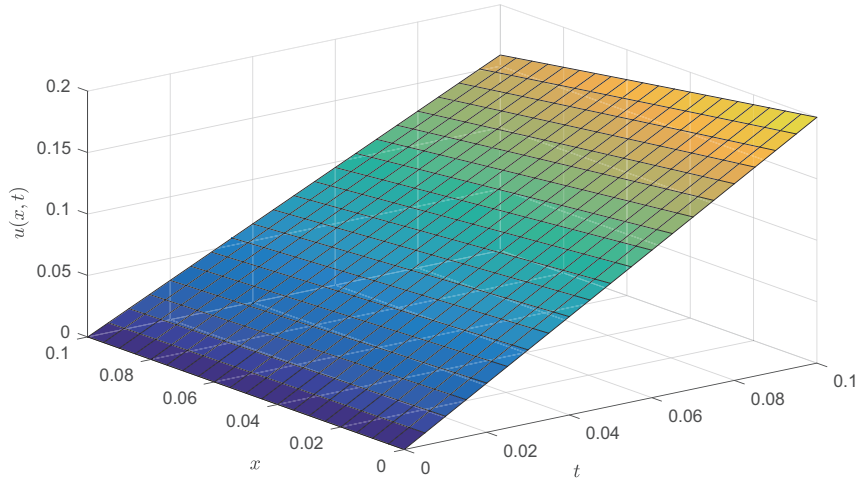

Figure 8: The RPS solution of Ex. 3 for $\alpha=1, \beta=1$.

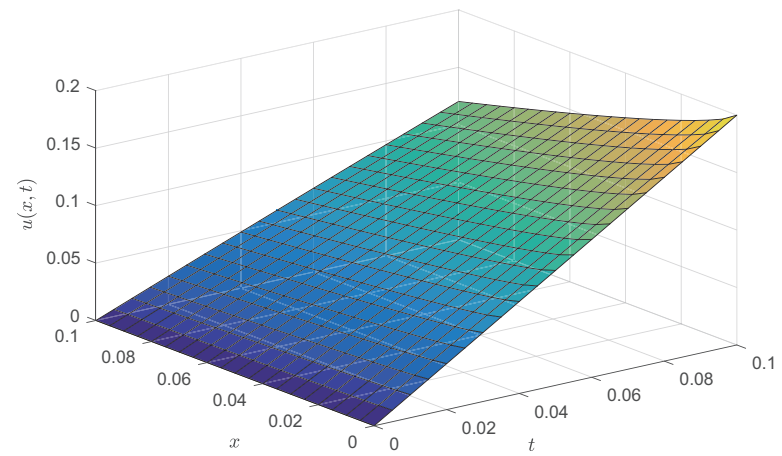

Figure 9: The RPS solution of Ex. 3 for $\alpha=0.75, \beta=0.75$. 
Table 3: The values of the approximate solutions for Example 3.

\begin{tabular}{|c|c|c|ccc|ccc|}
\hline$x$ & $t$ & Exact & $\beta=1$ & $\begin{array}{l}\alpha=1 \\
\beta=0.75\end{array}$ & $\beta=0.5$ & $\beta=1$ & $\beta=0.75$ & $\beta=0.5$ \\
\hline 0.2 & 0.03 & 0.37744 & 0.37569 & 0.37670 & 0.37848 & 0.33452 & 0.33708 & 0.34161 \\
& 0.06 & 0.35776 & 0.35074 & 0.35274 & 0.35626 & 0.28675 & 0.29089 & 0.29829 \\
& 0.09 & 0.34096 & 0.32517 & 0.32812 & 0.33334 & 0.24278 & 0.24820 & 0.25798 \\
0.4 & 0.03 & 0.75488 & 0.75078 & 0.75348 & 0.75740 & 0.66392 & 0.67095 & 0.68126 \\
& 0.06 & 0.71552 & 0.69912 & 0.70541 & 0.71238 & 0.55899 & 0.57076 & 0.58822 \\
& 0.09 & 0.68192 & 0.64503 & 0.65310 & 0.66493 & 0.45891 & 0.47480 & 0.49857 \\
0.6 & 0.03 & 1.13232 & 1.12534 & 1.12985 & 1.13567 & 0.98868 & 1.00080 & 1.01657 \\
& 0.06 & 1.07328 & 1.04537 & 1.05450 & 1.06632 & 0.81815 & 0.83904 & 0.86652 \\
& 0.09 & 1.02288 & 0.96009 & 0.97394 & 0.99195 & 0.65100 & 0.67993 & 0.71830 \\
0.8 & 0.03 & 1.50976 & 1.49943 & 1.50563 & 1.51290 & 1.30932 & 1.32646 & 1.34676 \\
& 0.06 & 1.43104 & 1.38972 & 1.40243 & 1.41739 & 1.06563 & 1.09598 & 1.13227 \\
& 0.09 & 1.36384 & 1.27087 & 1.29039 & 1.31348 & 0.82163 & 0.86459 & 0.91632 \\
\hline
\end{tabular}

Illustrative examples for Case 2 are given below:

Example 4. Let us consider the problem including FIE

$$
\begin{aligned}
D_{t}^{\alpha} u= & I_{x}^{\beta} u+2 u, \quad 0<\alpha \leq 1,1<\beta \leq 2, \\
u(x, 0) & =\exp (2 x), \\
u(0, t) & =\exp \left(\frac{t}{4}\right), \\
u_{x}(0, t) & =2 \exp \left(\frac{t}{4}\right),
\end{aligned}
$$

which has the exact solution $u(x, t)=\exp \left(2 x+\frac{t}{4}\right)$. Applying transformations to Eqs. (59)- (62), we have :

$$
\begin{aligned}
D_{t}^{\alpha}\left(D_{x}^{\beta} v\right)= & v-\frac{I_{x}^{\beta} 2 \exp \left(\frac{t}{4}\right) x}{\Gamma(3-\beta) \Gamma(\beta)}-\frac{I_{x}^{\beta} \exp \left(\frac{t}{4}\right)}{\Gamma(3-\beta) \Gamma(\beta-1)}+2 D_{x}^{\beta} v \\
& v(x, 0)=I_{x}^{\beta} \exp (2 x)=f_{0}(x) \\
& v(0, t)=I_{x}^{\beta} \exp \left(\frac{t}{4}\right) \\
& v_{x}(0, t)=2 I_{x}^{\beta} \exp \left(\frac{t}{4}\right)
\end{aligned}
$$


Table 4: The values of approximate solutions for Example 4.

\begin{tabular}{|c|c|c|c|c|c|c|c|c|}
\hline \multirow{2}{*}{$x$} & \multirow[t]{2}{*}{$t$} & \multirow{2}{*}{ Exact } & \multicolumn{3}{|c|}{$\alpha=1$} & \multicolumn{3}{|c|}{$\alpha=0.75$} \\
\hline & & & $\beta=2$ & $\beta=1.75$ & $\beta=1.5$ & $\beta=2$ & $\beta=1.75$ & $\beta=1.5$ \\
\hline \multirow[t]{3}{*}{0.2} & 0.3 & 1.60801 & 1.57778 & 1.58497 & 1.59779 & 1.62770 & 1.64272 & 1.66872 \\
\hline & 0.6 & 1.73325 & 1.67188 & 1.68847 & 1.71803 & 1.73424 & 1.76375 & 1.81483 \\
\hline & 0.9 & 1.86825 & 1.77412 & 1.80232 & 1.85256 & 1.83691 & 1.88195 & 1.95993 \\
\hline \multirow[t]{3}{*}{0.4} & 0.3 & 2.39888 & 2.34096 & 2.36367 & 2.39796 & 2.43343 & 527 & 175 \\
\hline & 0.6 & 2.58571 & 2.47337 & 2.52561 & 2.60440 & 2.60880 & 2.71025 & 2.85986 \\
\hline & 0.9 & 2.78710 & 2.62279 & 2.71136 & 2.84487 & 2.78498 & 2.93944 & 3.16717 \\
\hline \multirow[t]{3}{*}{0.6} & 0.3 & 3.57870 & 3.49656 & 3.54295 & 3.60693 & 3.70125 & 3.82151 & 3.98572 \\
\hline & 0.6 & 3.85743 & 3.70789 & 3.81421 & 3.96072 & 4.04099 & 4.27549 & 4.59551 \\
\hline & 0.9 & 4.15786 & 3.95409 & 4.13390 & 4.38151 & 4.39212 & 4.74827 & 5.23415 \\
\hline \multirow[t]{3}{*}{0.8} & 0.3 & 5.33880 & 5.23739 & 5.31775 & 5.42247 & 5.70419 & 5.95203 & 6.27766 \\
\hline & 0.6 & 5.75460 & 5.58752 & 5.77116 & 6.01022 & 6.39297 & 6.87479 & 7.50742 \\
\hline & 0.9 & 6.20280 & 6.00342 & 6.31327 & 6.71629 & 7.11462 & 7.84491 & 8.80337 \\
\hline
\end{tabular}

The $k^{\text {th }}$-truncated residual function becomes

$$
\operatorname{Res}_{k}=D_{t}^{\alpha}\left(D_{x}^{\beta} v_{k}\right)-v_{k}+\frac{I_{x}^{\beta} 2 \exp \left(\frac{t}{4}\right) x}{\Gamma(3-\beta) \Gamma(\beta)}+\frac{I_{x}^{\beta} \exp \left(\frac{t}{4}\right)}{\Gamma(3-\beta) \Gamma(\beta-1)}, k=0,1,2, \ldots
$$

Based on the RPS procedure, the first two coefficients are obtained as follows:

$$
\begin{aligned}
& f_{1}(x)=I_{x}^{\beta} \exp (2 x), \\
& f_{2}(x)=I_{x}^{2 \beta} \exp (2 x),
\end{aligned}
$$

and so on.

Hence the approximate solution of (59) lowing form:

$$
\begin{aligned}
u(x, t)= & \exp (2 x)+\frac{x^{2+\beta}}{4} E_{1,3+\beta}(2 x) \frac{t^{\alpha}}{\Gamma(1+\alpha)} \\
& +\frac{x^{2+2 \beta}}{16} E_{1,3+2 \beta}(2 x) \frac{t^{2 \alpha}}{\Gamma(1+2 \alpha)}+\cdots
\end{aligned}
$$

From Table 4 and Figures 10-12, as it can be seen from the table that for $\alpha=0.75$ and $\beta=1.5$ the solution is the best approximation of the exact solution of FIE with integer orders for all values of $x$ and $t$. 


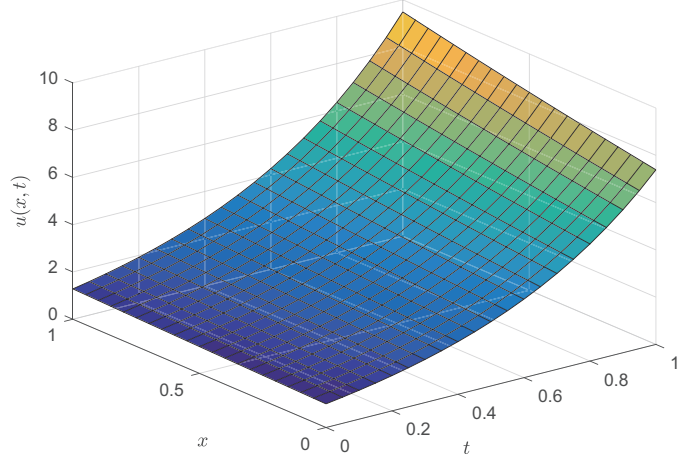

Figure 10: The exact solution of Ex. 4.

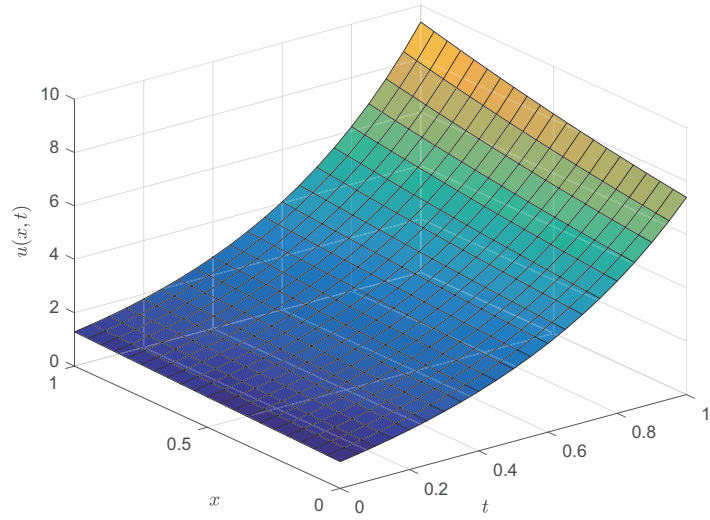

Figure 11: The RPS solution of Ex. 4 for $\alpha=1, \beta=2$.

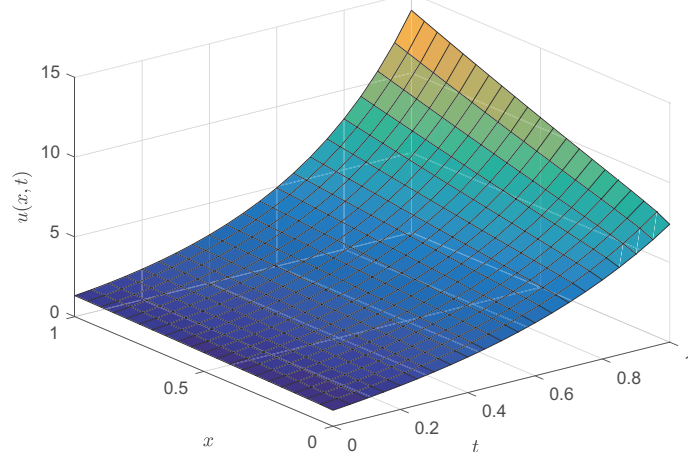

Figure 12: The RPS solution of Ex. 4 for $\alpha=0.75, \beta=1.75$. 
Example 5. Let us consider the problem including FIE

$$
\begin{aligned}
D_{t}^{\alpha} u= & I_{x}^{\beta} u+\left(1-\frac{x^{2}}{20}\right) u, 0<\alpha \leq 1,1<\beta \leq 2 \\
& u(x, 0)=20 x^{3} \\
& u(0, t)=0 \\
& u_{x}(0, t)=0
\end{aligned}
$$

which has the exact solution $u(x, t)=20 x^{3} \exp (t)$. Applying transformations to Eqs. (69) and (72), we have the followings:

$$
\begin{aligned}
D_{t}^{\alpha}\left(D_{x}^{\beta} v\right) & =v+\left(1-\frac{x^{2}}{20}\right) D_{x}^{\beta} v, \\
v(x, 0) & =I_{x}^{\beta}\left(20 x^{3}\right)=f_{0}(x), \\
v(0, t) & =0 \\
v_{x}(0, t) & =0 .
\end{aligned}
$$

The $k^{\text {th }}$-truncated residual function is

$$
\operatorname{Res}_{k}=D_{t}^{\alpha}\left(D_{x}^{\beta} v_{k}\right)-v_{k}-\left(1-\frac{x^{2}}{20}\right) D_{x}^{\beta} v_{k}, k=0,1,2, \ldots
$$

Based on the RPS procedure the first two coefficients are obtained as follows: $f_{1}(x)=I_{x}^{\beta}\left(20 x^{3}\right)+20 x^{3}-x^{5}$, $f_{2}(x)=I_{x}^{\beta}\left(f_{1}(x)+\left(1-\frac{x^{2}}{20}\right) D_{x}^{\beta} f_{1}(x)\right)$,

and so on.

Hence the approximate solution (69) -(172) is constructed in the following form:

$$
\begin{aligned}
u(x, t)= & 20 x^{3}+\left(20 \Gamma(4) \frac{x^{3+\beta}}{\Gamma(4+\beta)}+20 x^{3}-x^{5}\right) \frac{t^{\alpha}}{\Gamma(1+\alpha)} \\
& +\left(20 \Gamma(4) \frac{x^{3+2 \beta}}{\Gamma(4+2 \beta)}+20 \Gamma(4) \frac{x^{3+\beta}}{\Gamma(4+\beta)}-\Gamma(6) \frac{x^{5+\beta}}{\Gamma(6+\beta)}\right. \\
& \left.+\left(20-x^{2}\right) 6 \frac{x^{3+\beta}}{\Gamma(4+\beta)}+20 x^{3}-2 x^{3}+\frac{x^{7}}{20}\right) \frac{t^{2 \alpha}}{\Gamma(1+2 \alpha)}+\cdots
\end{aligned}
$$

In Table 5 and Figures $13-15$, as it is obvious that for $\alpha=1$ and $\beta=1.5$ the solution is the best approximation of the exact solution of FIE with integer orders for all values of $x$ and $t$. 


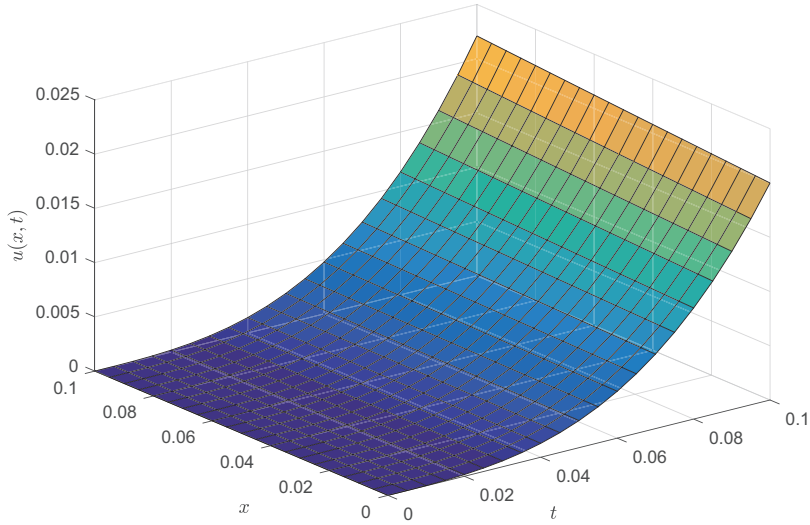

Figure 13: The exact solution of Ex. 5 .

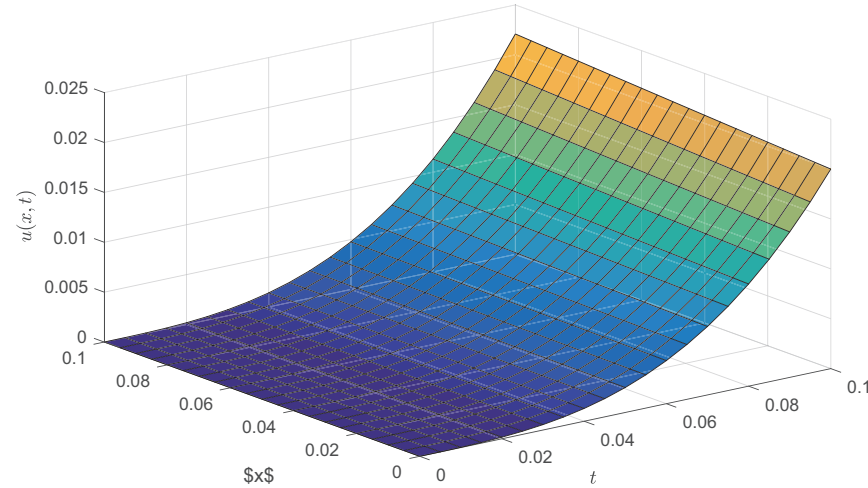

Figure 14: The RPS solution of Ex. 5 for $\alpha=1, \beta=2$.

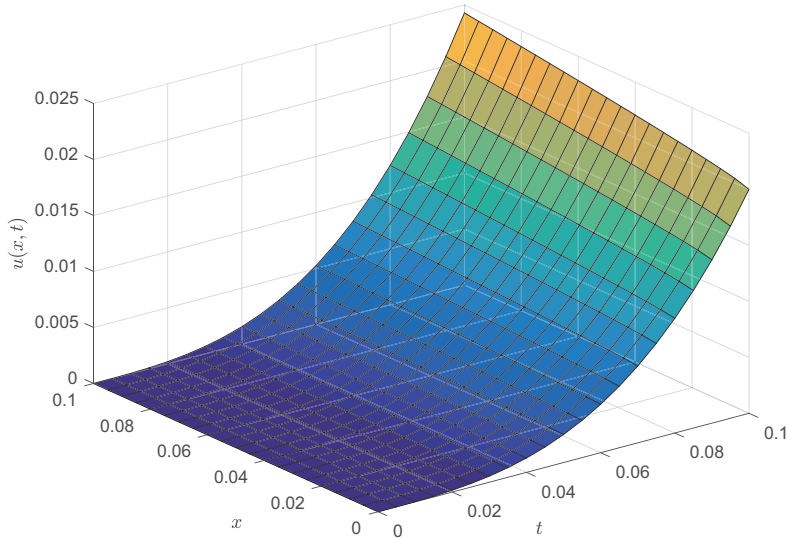

Figure 15: The RPS solution of Ex. 5 for $\alpha=0.75, \beta=1.75$. 
Table 5: The values of approximate solutions for Example 5.

\begin{tabular}{|c|c|l|lll|lll|}
\hline$x$ & $t$ & Exact & $\beta=2$ & $\begin{array}{l}\alpha=1 \\
\beta=1.75\end{array}$ & $\beta=1.5$ & $\beta=2$ & $\beta=1.75$ & $\beta=1.5$ \\
\hline 0.2 & 0.3 & 0.21598 & 0.21451 & 0.21467 & 0.21502 & 0.24845 & 0.24873 & 0.24936 \\
& 0.6 & 0.29154 & 0.28204 & 0.28243 & 0.28330 & 0.32925 & 0.32984 & 0.33116 \\
& 0.9 & 0.39354 & 0.36258 & 0.36328 & 0.36484 & 0.41376 & 0.41470 & 0.41679 \\
0.4 & 0.3 & 1.72782 & 1.71676 & 1.72042 & 1.72726 & 1.98948 & 1.99593 & 2.00802 \\
& 0.6 & 2.33231 & 2.25905 & 2.26805 & 2.28490 & 2.63938 & 2.65289 & 2.67822 \\
& 0.9 & 3.14829 & 2.90685 & 2.92288 & 2.95294 & 3.31997 & 3.34144 & 3.38172 \\
0.6 & 0.3 & 5.83139 & 5.79796 & 5.82012 & 5.85751 & 6.72518 & 6.76429 & 6.83040 \\
& 0.6 & 7.87155 & 7.63983 & 7.69438 & 7.78661 & 8.93812 & 9.02000 & 9.15866 \\
& 0.9 & 10.62549 & 9.84563 & 9.94280 & 10.10731 & 11.26040 & 11.39053 & 11.61110 \\
0.8 & 0.3 & 13.82255 & 13.75621 & 13.83423 & 13.95653 & 15.97661 & 16.11430 & 16.33068 \\
& 0.6 & 18.65850 & 18.16084 & 18.35289 & 18.65479 & 21.28689 & 21.57515 & 22.02929 \\
& 0.9 & 25.18634 & 23.45390 & 23.79597 & 24.33477 & 26.87547 & 27.33355 & 28.05632 \\
\hline
\end{tabular}

Example 6. Let us consider the problem including FIE

$$
\begin{aligned}
D_{t}^{\alpha} u= & I_{x}^{\beta} u+u u_{x}-\frac{24 x}{(1-2 t)^{2}}-\frac{x^{3}}{(1-2 t)}, \quad 0<\alpha \leq 1,1<\beta \leq 2 \\
& u(x, 0)=6 x \\
& u(0, t)=0 \\
& u_{x}(0, t)=\frac{6}{(1-2 t)},
\end{aligned}
$$

which has the exact solution $u(x, t)=\frac{6 x}{1-2 t}$. Applying transformations to Eqs. (79) and (82), we have the followings:

$$
\begin{aligned}
D_{t}^{\alpha}\left(D_{x}^{\beta} v\right)= & v+D_{x}^{\beta} v D_{x}^{\beta+1} v-\frac{24 x}{(1-2 t)^{2}}-\frac{x^{3}}{(1-2 t)}-\frac{I_{x}^{\beta} \frac{6}{(1-2 t)} x}{\Gamma(3-\beta) \Gamma(\beta)} \\
& v(x, 0)=6 \frac{x^{1+\beta}}{\Gamma(2+\beta)}=f_{0}(x) \\
& v(0, t)=0 \\
& v_{x}(0, t)=I_{x}^{\beta} \frac{6}{(1-2 t)}
\end{aligned}
$$

The $k^{t h}$-truncated residual function is

$$
\begin{aligned}
\operatorname{Res}_{k}= & D_{t}^{\alpha}\left(D_{x}^{\beta} v_{k}\right)-v_{k}-D_{x}^{\beta} v_{k} D_{x}^{\beta+1} v_{k} \\
& -\frac{24 x}{(1-2 t)^{2}}-\frac{x^{3}}{(1-2 t)}-\frac{I_{x}^{\beta} \frac{6}{(1-2 t)} x}{\Gamma(3-\beta) \Gamma(\beta)}, k=0,1,2, \ldots
\end{aligned}
$$


Based on the RPS procedure the first two coefficients are obtained as follows:

$$
\begin{aligned}
f_{1}(x)= & 6 \frac{x^{1+2 \beta}}{\Gamma(2+2 \beta)}+12 \frac{x^{1+\beta}}{\Gamma(2+\beta)}-6 \frac{x^{3+\beta}}{\Gamma(4+\beta)} \\
f_{2}(x)= & 6 \frac{x^{1+3 \beta}}{\Gamma(2+3 \beta)}+48 \frac{x^{1+2 \beta}}{\Gamma(2+2 \beta)}-6 \frac{x^{3+2 \beta}}{\Gamma(4+2 \beta)}+36 \frac{\Gamma(2+\beta)}{\Gamma(1+\beta)} \frac{x^{1+2 \beta}}{\Gamma(2+2 \beta)} \\
& +72 \frac{x^{1+\beta}}{\Gamma(2+\beta)}+144 \frac{x^{2+\beta}}{\Gamma(3+\beta)}-36 \frac{x^{3+\beta}}{\Gamma(4+\beta)}-144 \frac{x^{4+\beta}}{\Gamma(5+\beta)},
\end{aligned}
$$

and so on.

Hence the approximate solution (779) - (82) is constructed in the following form:

$$
\begin{aligned}
u(x, t)= & 6 x+\left(6 \frac{x^{1+\beta}}{\Gamma(2+\beta)}+12 x-x^{3}\right) \frac{t^{\alpha}}{\Gamma(1+\alpha)} \\
& +\left(6 \frac{x^{1+2 \beta}}{\Gamma(2+2 \beta)}+48 \frac{x^{1+\beta}}{\Gamma(2+\beta)}-6 \frac{x^{3+\beta}}{\Gamma(4+\beta)}\right. \\
& \left.+36 \frac{x^{1+\beta}}{\Gamma(2+\beta)}+72 x+72 x^{2}-6 x^{3}-6 x^{4}\right) \frac{t^{2 \alpha}}{\Gamma(1+2 \alpha)}+\cdots
\end{aligned}
$$

In Table 6 and Figures $16-18$, as it is obvious that for $\alpha=1$ and $\beta=2$ the solution is the best approximation of the exact solution of FIE with integer orders for all values of $x$.

Table 6: The values of approximate solutions for Example 6 .

\begin{tabular}{|c|c|c|ccc|ccc|}
\hline$x$ & $t$ & \multirow{2}{*}{ Exact } & \multicolumn{3}{|c|}{$\alpha=1$} & & & $\alpha=0.75$ \\
& & & $\beta=2$ & $\beta=1.75$ & $\beta=1.5$ & $\beta=2$ & $\beta=1.75$ & $\beta=1.5$ \\
\hline 0.2 & 0.03 & 1.27660 & 1.27984 & 1.28018 & 1.28081 & 1.45637 & 1.45776 & 1.46037 \\
& 0.06 & 1.36364 & 1.37537 & 1.37621 & 1.37780 & 1.70928 & 1.71247 & 1.71841 \\
& 0.09 & 1.46341 & 1.48659 & 1.48810 & 1.49095 & 1.98312 & 1.98844 & 1.99833 \\
0.4 & 0.03 & 2.55319 & 2.56265 & 2.56446 & 2.56736 & 2.93848 & 2.94598 & 2.95773 \\
& 0.06 & 2.72727 & 2.76260 & 2.76713 & 2.77430 & 3.49138 & 3.50852 & 3.53514 \\
& 0.09 & 2.92683 & 2.99986 & 3.00802 & 3.02084 & 4.09999 & 4.12861 & 4.17285 \\
0.6 & 0.03 & 3.82979 & 3.84870 & 3.85335 & 3.86001 & 4.44874 & 4.46786 & 4.49467 \\
& 0.06 & 4.09091 & 4.16279 & 4.17439 & 4.19081 & 5.35307 & 5.39664 & 5.45715 \\
& 0.09 & 4.39024 & 4.54228 & 4.56312 & 4.59239 & 6.36310 & 6.43571 & 6.53604 \\
0.8 & 0.03 & 5.10638 & 5.13816 & 5.14694 & 5.15854 & 5.98864 & 6.02444 & 6.07065 \\
& 0.06 & 5.45455 & 5.57663 & 5.59843 & 5.62685 & 7.29862 & 7.37988 & 7.48369 \\
& 0.09 & 5.85366 & 6.11542 & 6.15446 & 6.20495 & 8.78025 & 8.91540 & 9.08712 \\
\hline
\end{tabular}




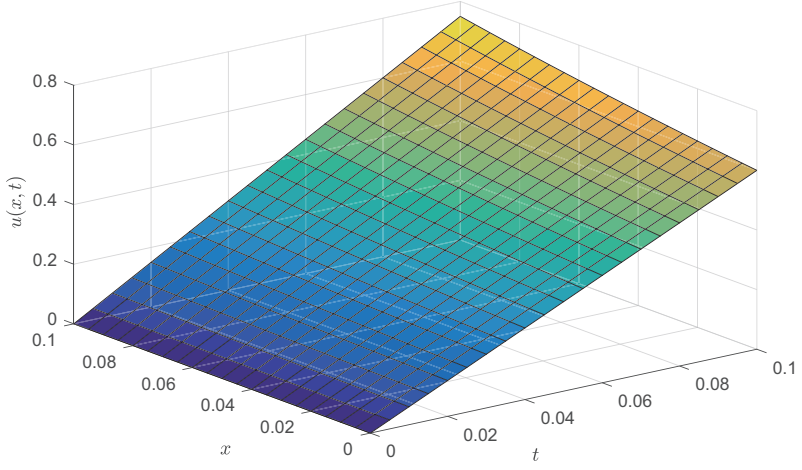

Figure 16: The exact solution of Ex. 6 .

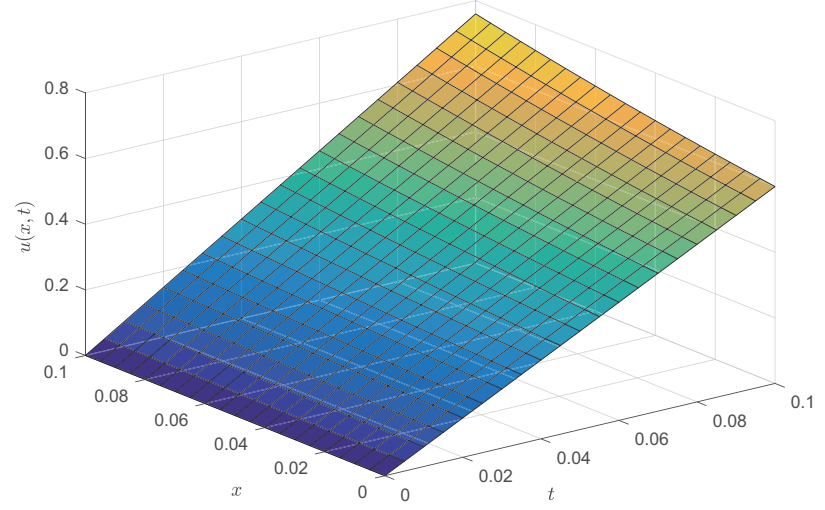

Figure 17: The RPS solution of Ex. 6 for $\alpha=1, \beta=2$.

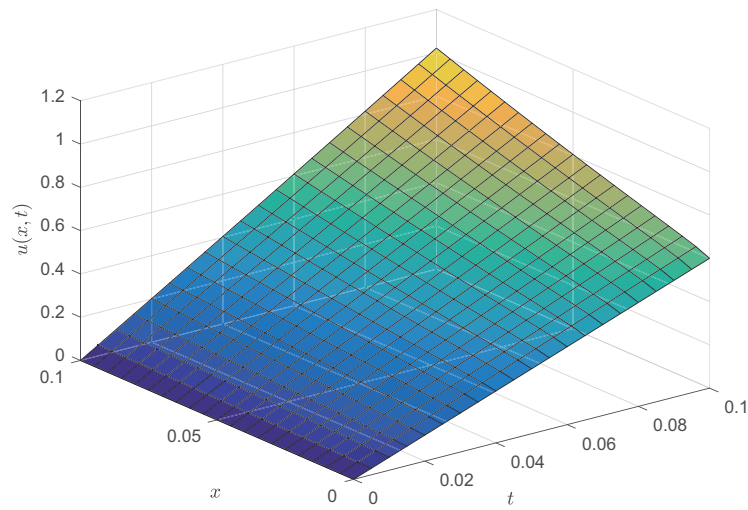

Figure 18: The RPS solution of Ex. 6 for $\alpha=0.75, \beta=1.75$. 


\section{Conclusion}

In order to obtain the analytic solution of FIE, the RPSM is used with new transformation which makes the implementation of RPSM easier. Examples for different values of $\alpha$ and $\beta$ illustrate that this method is feasible and convenient to establish the analytic solution for this kind of problems.

\section{References}

1. S. Abbasbandy, The application of homotopy analysis method to nonlinear equations arising in heat transfer, Phys. Lett. A, 360(2006), 109-113.

DOI:10.1016/j.physleta.2006.07.065.

2. O. Abu-Arqub, Series solution of fuzzy differential equations under strongly generalized differentiability. The J. of Adv. Res. in Appl. Math., 5(2013), 31-52. DOI:10.5373/jaram.1447.051912.

3. O. Abu-Arqub, Z. Abo-Hammour, R. Al-Badarneh, and S. Momani, A reliable analytical method for solving higher-order initial value problems, Discr. Dyn. in Nat. and Soc., 12(2013). DOI:10.1155/2013/673829.

4. O. Abu-Arqub, A. El-Ajou, Z. Al-Zhour and S. Momani, Multiple solutions of nonlinear boundary value problems of fractional order: A New Analytic Iterative Technique, Entropy., 16(2014), 471-493. DOI:10.3390/e16010471.

5. Z. Ahmad and I. Fino Hassan, Analytical solution for a generalized space-time fractional telegraph equation, Math. Meth. in the Appl. Sci., 36(2013), 14. DOI:10.1002/mma.2727.

6. O. Abu-Arqub, A. El-Ajou and S. Momani, Constructing and predicting solitary pattern solutions for nonlinear time-fractional dispersive partial differential equations, $J$. of Comput Phys., 293(2015), 385-399. DOI:10.1016/j.jcp.2014.09.034.

7. A. Atangana, On the new fractional derivative and application to nonlinear Fisherreaction-diffusion equation, Appl. Math. and Comput., 273(2016), 948-956. DOI: 10.1016/j.amc.2015.10.021.

8. A. Atangana and D. Baleanu, New fractional derivatives with non-local and nonsingular kernel: Theory and application to heat transfer model, Thermal Sci., 20(2016), 763-769. DOI:10.2298/TSCI160111018A.

9. A. Atangana and I. Koca, New direction in fractional differentiation, Math. in Nat. Sci., 1(2017), 18-25. DOI:10.22436/mns.01.01.02.

10. J.-S. Duan, R. Rach, D. Baleanau, A.-M. Wazwaz, A review of the Adomian decomposition method and its applications to fractional differential equations, Commun in Fract Calc., 3(2012), 73-99.

11. J. Hristov, The non-linear Dodson diffusion equation: Approximate solutions and beyond with formalistic fractionalization, Math. in Nat. Sci., (2017), 1-17. DOI:10.22436/mns.01.01.01. 
12. Y. Jalilian and M. Ghasemi, On the Solutions of a Nonlinear Fractional IntegroDifferential Equation of Pantograph, Mediterr J. Math., 14(2017), 194. DOI: 10.1007/s00009-017-0993-8.DOI:10.1007/s00009-017-0993-8.

13. A. El-Ajou, O. Abu-Arqub, Z. Al-Zhour and S. Momani, New results on fractional power series: theories and applications, Entropy., 15(2013), 5305-5323. DOI:10.3390/e15125305.

14. A. El-Ajou, O. Abu-Arqub, S. Momani, D. Baleanu and A. Alsaedi, A novel expansion iterative method for solving linear partial differential equations of fractional order, Appl. Math. and Comput., 257(2015), 119-133. DOI:10.1016/j.amc.2014.12.121.

15. A.-A. Kilbas, H.-M. Srivastava, J.-J. Trujillo, Theory and applications of fractional differential equations, Amsterdam: Elsevier, 2006.

16. S. Kumar, A new analytical modelling for telegraph equation via Laplace transform, Appl. Math. Model., 38(2014), No.13, 3154-3163. DOI:10.1016/j.apm.2013.11.035.

17. S. Kumar, A numerical study for solution of time fractional nonlinear shallowwater equation in Oceans, Zeitschrift fur Naturforschung A., 68(a)(2013), 1-7. DOI: 10.5560/ZNA.2013-0036.

18. F. Mirzaee and S. Alipour, Fractional-order orthogonal Bernstein polynomials for numerical solution of nonlinear fractional partial Volterra integro-differential equations, Math. Meth. in the Appl. Sci., 42(2019), 6. DOI:10.1002/mma.5481.

19. S. Momani and Z. Odibat, Analytical solution of a time-fractional Navier-Stokes equation by Adomian decomposition method, Appl. Math. and Comput., 177(2006), 488494. DOI:10.1016/j.amc.2005.11.025.

20. S. Odibat and S. Momani, Application of variational iteration method to nonlinear differential equations of fractional order, Intern. J. of Nonlin. Sci. and Num. Simul., 7(2006), 27-34. DOI:10.1515/IJNSNS.2006.7.1.27.

21. I. Podlubny, Fractional Differential Equation, San Diego, CA: Academic Press, 1999.

22. J. Sabatier, O.-P. Agarwal, and J.A.T. Machado (eds). Advances in Fractional Calculus: Theoretical Developments and Applications in Physics and Engineering, Dordrecht: Springer, 2007.

23. S.-G. Samko, A.-A. Kilbas and O.-I. Marichev, Fractional Integrals and Derivatives Theory and Applications, Amsterdam: Gordon and Breach, 1993.

24. D.-L. Suthar, S.-D. Purohit and R.-K. Parmar, Generalized fractional calculus of the multi-index Bessel function, Math. in Nat. Sci., 1(2017), 26-32.

DOI:10.22436/mns.01.01.03.

25. X.-J. Yang and D. Baleanu, Fractal heat conduction problem solved by local fractional variational iteration method, Thermal Science, 17(2013), 625-628. DOI:10.2298/TSCI121124216Y.

26. X. Zhang, J. Zhao, J. Liu and B. Tang, Homotopy perturbation method for two dimensional time-fractional wave equation, Appl. Math. Model., 38(2014), 5545-5552. DOI:10.1016/j.apm.2014.04.018.

27. A.-H. Ahmed, P. G. Kirtiwant and M. A. Shakir, The approximate solutions of fractional integro-differential equations by using modified Adomian decomposition method, Khayyam J. Math., 5 (2019), No.1, 21-39. DOI:10.22034/kjm.2018.73593. 